\title{
Roles of the Bloom's syndrome helicase in the maintenance of genome stability
}

\author{
C.F. Cheok, C.Z. Bachrati, K.L. Chan, C. Ralf, L. Wu and I.D. Hickson ${ }^{1}$ \\ Cancer Research UK Laboratories, Weatherall Institute of Molecular Medicine, University of Oxford, John Radcliffe Hospital, Oxford OX3 9DS, U.K.
}

\begin{abstract}
The RecQ family of DNA helicases is highly conserved in evolution from bacteria to humans. Of the five known human RecQ family members, three (BLM, WRN and RECQ4, which cause Bloom's syndrome, Werner's syndrome and Rothmund-Thomson syndrome respectively) are mutated in distinct clinical disorders associated with cancer predisposition and/or premature aging. BLM forms part of a multienzyme complex including topoisomerase Ill $\alpha$, replication protein A and a newly identified factor called BLAP75. Together, these proteins play a role in the resolution of DNA structures that arise during the process of homologous recombination repair. In the absence of BLM, cells show genomic instability and a high incidence of sisterchromatid exchanges. In addition to a DNA structure-specific helicase activity, BLM also catalyses Hollidayjunction branch migration and the annealing of complementary single-stranded DNA molecules.
\end{abstract}

\section{Introduction}

BS (Bloom's syndrome) is an autosomal recessive disorder characterized by growth retardation, sunlight sensitivity and a predisposition to the development of cancer [1]. At the cellular level, BS is associated with inherent genomic instability. In comparison with cells isolated from unaffected individuals, BS cells show an elevated frequency of several types of chromosomal aberrations, including breaks, quadriradials and translocations [1-3]. The hallmark feature, which is sufficiently specific to BS cells to be used in the molecular diagnosis of the disorder, is an approx. 10-fold elevation in the frequency of SCEs (sister-chromatid exchanges) [4].

BLM (the BS gene product) is one of five RecQ helicase family members expressed in human cells $[2,3]$. The RecQ family is highly conserved in evolution from bacteria to humans, particularly in the centrally-located helicase domain that contains seven characteristic sequence motifs. BLM, in common with Escherichia coli RecQ and its Saccharomyces cerevisiae orthologue, Sgs1p, contains two additional conserved domains. These domains, which are located C-terminal to the helicase domain, are the RQC (RecQ Cterminal) domain and the HRDC (helicase and RNAse D C-terminal) domain. Biochemical and structural analyses have indicated that the RQC and HRDC domains probably function as auxiliary DNA-binding sites [5-7].

BLM interacts physically and functionally with a number of other nuclear factors in human cells. Most notably, BLM binds directly to topoisomerase III $\alpha$, to the RAD51 recombinase, and to RPA [replication protein A, the major ssDNA (single-stranded DNA)-binding protein in human cells] [8-10]. Significantly, these interactions are conserved in

Key words: BLAP75, BLM, RecQ helicase, sister-chromatid exchange, strand annealing, topoisomerase III.

Abbreviations used: BLM, Bloom's syndrome gene product; BS, Bloom's syndrome; HRDC, helicase and RNAse D C-terminal; RPA, replication protein A; RQC, RecQ C-terminal; SCE, sisterchromatid exchange; ss, single stranded; Top3p, topoisomerase III protein.

${ }^{1}$ To whom correspondence should be addressed (email ian.Hickson@cancer.org.uk). evolution; for example, Sgs1p forms a complex with yeast Top3p (topoisomerase III protein) and Rad51p [10,11]. The functional significance of these interactions has been highlighted by the fact that RPA strongly stimulates BLM helicase activity and, in turn, BLM stimulates topoisomerase III $\alpha$ to relax negatively-supercoiled DNA $[8,12]$.

Recently, a functionally important binding partner of RecQ helicases was identified independently in both yeast and human cells. In yeast, large-scale genetic screens identified RMI1 (NCE4) as a gene that interacts genetically with $S G S 1$ and, when mutated, produces a phenotype very similar to that of top3 $\Delta$ strains $[13,14]$. Subsequent work indicated that Sgs1p, Top3p and Rmi1p form a complex in yeast cells $[13,14]$. The human Rmi1 orthologue, named BLAP75, was identified as a protein that co-immunoprecipitates with BLM [15]. Crucially, it was shown that siRNA (small interfering RNA)-mediated depletion of cellular BLAP75 led to an increase in SCEs, indicating a functional overlap between BLM and BLAP75.

\section{BLM is a DNA structure-specific helicase}

In the cases where they have been analysed, RecQ helicases catalyse DNA unwinding, i.e. the separation of the complementary strands of duplex DNA. As with most helicases, RecQ family proteins translocate unidirectionally along DNA ( $3^{\prime} \rightarrow 5^{\prime}$ for RecQ proteins), which is defined by the orientation of the strand to which the enzyme is bound, not the direction of displacement of the complementary strand [16].

One striking feature of RecQ helicases is their ability to recognize and unwind a wide variety of different DNA structures (Figure 1). In the case of BLM, this includes forked duplexes, synthetic four-way junctions that mimic the Holliday-junction recombination-intermediate, and bluntended duplexes with an internal 'bubble' of non-complementary sequence [17]. Interestingly, a fully double-stranded, 
Figure 1 Examples of the range of DNA structures that can be unwound by the BLM helicase

(A) 3'-Tailed duplex, (B) 'bubble' substrate, (C) forked duplex, (D) synthetic 4-way (Holliday) junction, (E) synthetic displacement loop, (F) G-quadruplex structure.
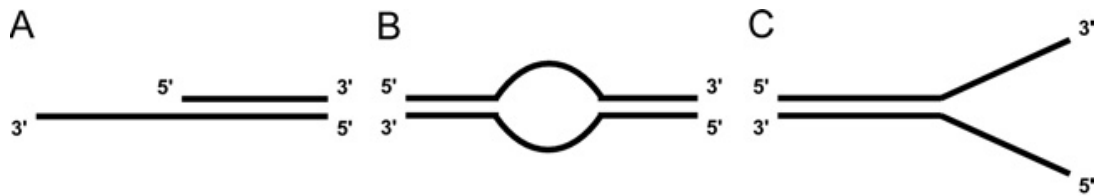

D

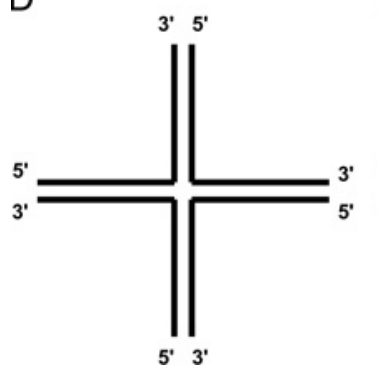

E

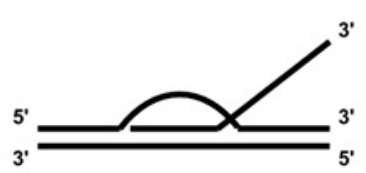

$\mathrm{F}$

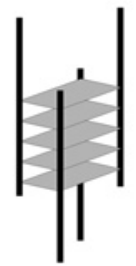

blunt-ended structure is not a substrate. BLM, like other RecQ helicases, also shows a preference for unwinding Gquadruplex DNA [18]. G-quadruplexes are highly stable, non-B-form DNA structures that can form in guaninerich DNA sequences [19]. The functional significance of G-quadruplex unwinding by BLM remains unknown, but a possible role in disruption of telomeric quadruplexes is suggested by the fact that BLM and other RecQ helicases are known to influence telomere stability under certain circumstances [2,3]. As far as we are aware, RecQ helicases are unique among helicases in being able to disrupt G-quadruplexes efficiently.

\section{BLM catalyses DNA strand annealing}

BLM utilizes the energy derived from the hydrolysis of ATP to catalyse DNA strand separation. During an analysis of the effects of varying BLM concentration on the extent of DNA unwinding, we consistently observed that high concentrations of BLM were apparently less active than were lower BLM concentrations. As a result, when the level of the unwound reaction product was plotted as a function of BLM concentration, a pronounced 'bell-shaped' curve was generated (Figure 2A). We considered it possible that this effect reflected a propensity of BLM to aggregate at high concentrations. However, an alternative explanation for the reappearance of the duplex DNA substrate in reactions containing high concentrations of BLM (Figure 2A) was that BLM could catalyse the 'reverse' reaction to that of a helicase; namely, the annealing of the complementary ssDNA reaction products. To analyse this directly, we incubated various concentrations of BLM with the two partially complementary ssDNAs (unannealed) used in Figure 2(A) to create the forked duplex substrate. As indicated in Figure 2(B), there was a BLM concentration-dependent increase in the annealing of the ssDNA to form a forked duplex. This DNA strand-
Figure 2 | BLM catalyses ssDNA annealing

(A) Effect of increasing BLM concentration on the conversion of a forked duplex to ssDNA (as indicated on the left). Data are quantified in the panel on the right. (B) BLM promotes annealing of complementary ssDNA. Left panel shows a BLM concentration-dependent increase in the level of annealed forked-duplex-DNA. Right panel shows that truncation of the C-terminal domain of BLM by 127 amino acids to create BLM-(1-1290) inactivates the strand-annealing function.

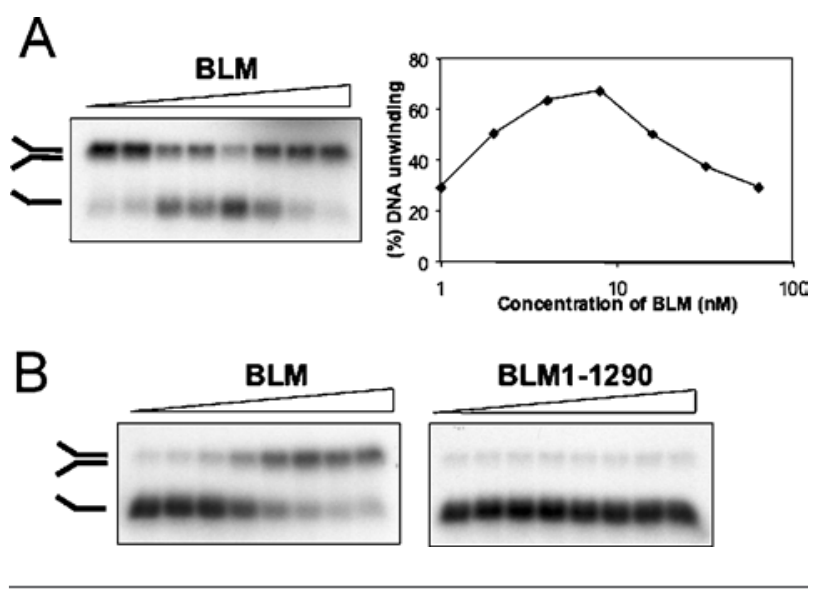

annealing activity of BLM is ATP-independent and can be blocked by ssDNA-binding proteins such as RPA [20].

As part of an analysis of the domains within BLM that are required for the different enzymatic functions of the protein, we have shown that the C-terminal domain of BLM is essential for ssDNA annealing. For example, BLM-(1-1290) protein, which lacks the C-terminal 127 amino acids of BLM, is unable to catalyse strand annealing (Figure 2B). Additional analyses identified a 50-amino-acid region of BLM between residues 1290 and 1350 as being essential for promoting strand annealing [20]. At this stage, we do not understand the precise mechanism by which this activity of BLM is effected. 
The C-terminal truncation of BLM probably influences the integrity of the HRDC domain, which is predicted to be a DNA-binding motif in BLM. Two obvious models for the mechanism of strand annealing are: (i) that BLM brings two ssDNA molecules into close proximity via protein-protein interactions, with each BLM protomer containing a ssDNA species bound via its HRDC domain; or (ii) that through making specific DNA contacts on each of the ssDNA species, BLM could limit or neutralize charge repulsion and hence facilitate base-pairing. Further work is required to assess whether either of these models is correct.

\section{BLM catalyses a novel mechanism for resolution of recombination intermediates}

The hallmark feature of BS cells is an unusually high frequency of SCEs [4]. These apparently reciprocal exchanges arise primarily as part of homologous recombination events that occur during repair of DNA damage arising in the $S$ or $G_{2}$ phases of the cell cycle. This, together with many other strands of evidence, such as the finding that BLM interacts with the RAD51 recombinase and the RAD51 paralogue, RAD51D [10,21], indicates that BLM somehow influences recombination reactions.

Our group has focused on the idea that the combination of a RecQ helicase and a type IA topoisomerase (topoisomerase III $\alpha$ in humans) is involved in resolution of recombination intermediates that contain Holliday junctions. It has long been considered whether topoisomerases might catalyse Holliday-junction resolution via their ability to cut DNA strands and pass other strands through the break. Nevertheless, we and others were unsuccessful in our attempts to demonstrate any selective cleavage of Holliday-junction cross-overs by topoisomerase III enzymes. Many models of recombination involve the processing of DNA lesions in such a way as to generate a so-called double Holliday junction, in which two junctions lie in relatively close proximity to one another. One proposal set out previously [22] was that a helicase could migrate these junctions towards one another to form a single intertwined species called a hemicatenane and that topoisomerase III would 'resolve' this by ss passage (decatenation) to generate recombinant products (Figure 3 ). The beauty of this model is that it avoids any formation of socalled cross-over recombinant products, which are visualized cytologically as SCEs, and hence would fit neatly with a role for BLM in suppression of SCE formation.

Recently, we provided direct experimental evidence for the above model in demonstrating that BLM and topoisomerase III $\alpha$ catalyse a novel reaction for the resolution of double Holliday junctions, termed Holliday junction dissolution [23]. This reaction requires the hydrolysis of ATP by BLM and the active site tyrosine residue of topoisomerase III $\alpha$. Although the full details of the reaction mechanism underlying junction dissolution remain to be elucidated, the reaction gives rise exclusively to non-cross-over products, as predicted from the hemicatenane model (Figure 3 ) and the role of BLM as a suppressor of SCEs. In addition to the
Figure 3 Model for the resolution of double Holliday junctions without crossing-over

The two recombining molecules are coloured grey and black. The double Holliday junction (top) is converted by branch migration of the junctions to a hemicatenane (middle). The hemicatenane is the substrate for the ssDNA decatenation (strand passage) activity of topoisomerase III. The products (bottom) are resolved DNA molecules with no crossing-over of the flanking DNA. See text for details.

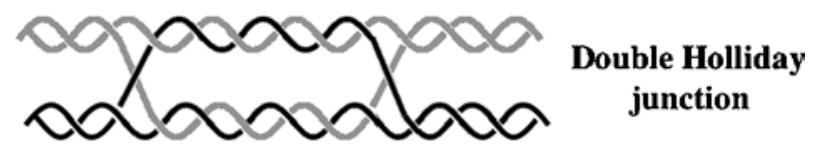

Branch migration

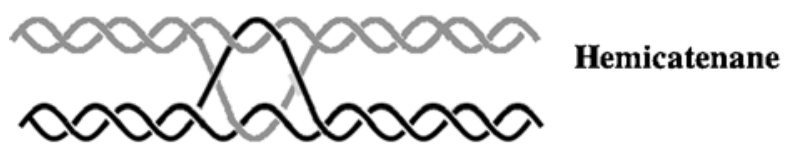

Decatenation
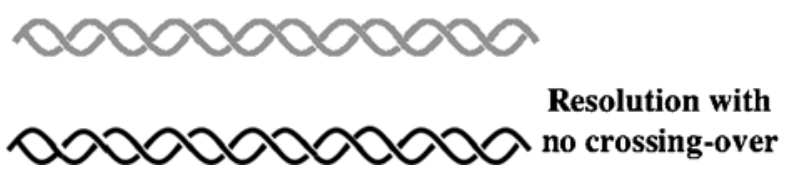

helicase domain, we have shown that the HRDC domain of BLM is essential for dissolution. As part of these studies, we identified Lys ${ }^{1270}$, which is predicted to contact DNA on the DNA-binding face of the HRDC domain, as being important for efficient dissolution [24].

In summary, BLM forms part of a multienzyme complex that appears to play roles both in the disruption of alternative DNA structures, such as quadruplexes, and in the resolution of DNA intermediates that arise during homologous-recombination reactions. Given the similarity between these recombination intermediates and DNA structures that are predicted to arise during the late stages of DNA replication where two forks converge, it will be interesting to analyse whether BLM and topoisomerase III $\alpha$ also play a role in resolution of replication structures. Another new challenge is to define the biochemical role of the BLAP75 subunit of the BLM complex.

We thank the members of the Cancer Research UK Genome Integrity and Chromosome Stability laboratories for ongoing discussions. Work in the authors' laboratory is supported by Cancer Research UK. K.L.C. is supported by the Croucher Foundation (Hong Kong).

\section{References}

1 German, J. (1995) Dermatol. Clin. 13, 7-18

2 Bachrati, C.Z. and Hickson, I.D. (2003) Biochem. J. 374, 577-606

3 Hickson, I.D. (2003) Nat. Rev. Cancer 3, 169-178 
4 Chaganti, R.S., Schonberg, S. and German, J. (1974) Proc. Natl. Acad. SCi. U.S.A. 71, 4508-4512

5 Morozov, V., Mushegian, A.R., Koonin, E.V. and Bork, P. (1997) Trends Biochem. Sci. 22, 417-418

6 Liu, Z., Macias, M.J., Bottomley, M.J., Stier, G., Linge, J.P., Nilges, M., Bork, P. and Sattler, M. (1999) Structure (London) 7, 1557-1566

7 Bernstein, D.A., Zittel, M.C. and Keck, J.L. (2003) EMBO J. 22, 4910-4921

8 Brosh, Jr, R.M., Li, J.L., Kenny, M.K., Karow, J.K., Cooper, M.P., Kureekattil, R.P., Hickson, I.D. and Bohr, V.A. (2000) J. Biol. Chem. 275, 23500-23508

9 Wu, L., Davies, S.L., North, P.S., Goulaouic, H., Riou, J.F., Turley, H., Gatter, K.C. and Hickson, I.D. (2000) J. Biol. Chem. 275, 9636-9644

10 Wu, L., Davies, S.L., Levitt, N.C. and Hickson, I.D. (2001) J. Biol. Chem. 276, 19375-19381

11 Gangloff, S., McDonald, J.P., Bendixen, C., Arthur, L. and Rothstein, R. (1994) Mol. Cell. Biol. 14, 8391-8398

12 Wu, L. and Hickson, I.D. (2002) Nucleic Acids Res. 30, 4823-4829

13 Mullen, J.R., Nallaseth, F.S., Lan, Y.Q., Slagle, C.E. and Brill, S.J. (2005) Mol. Cell. Biol. 25, 4476-4487

14 Chang, M., Bellaoui, M., Zhang, C., Desai, R., Morozov, P., Delgado-Cruzata, L., Rothstein, R., Freyer, G.A., Boone, C. and Brown, G.W. (2005) EMBO J. 24, 2024-2033
15 Yin, J., Sobeck, A., Xu, C., Meetei, A.R., Hoatlin, M., Li, L. and Wang, W. (2005) EMBO J. 24, 1465-1476

16 Soultanas, P. and Wigley, D.B. (2001) Trends Biochem. Sci. 26, 47-54

17 Mohaghegh, P., Karow, J.K., Brosh, Jr, R.M., Bohr, V.A. and Hickson, I.D. (2001) Nucleic Acids Res. 29, 2843-2849

18 Sun, H., Karow, J.K., Hickson, I.D. and Maizels, N. (1998) J. Biol. Chem. 273, 27587-27592

19 Williamson, J.R. (1994) Annu. Rev. Biophys. Biomol. Struct. 23, 703-730

20 Cheok, C.F., Wu, L., Garcia, P.L., Janscak, P. and Hickson, I.D. (2005) Nucleic Acids Res. 33, 3932-3941

21 Braybrooke, J.P., Li, J.L., Wu, L., Caple, F., Benson, F.E. and Hickson, I.D (2003) J. Biol. Chem. 278, 48357-48366

22 Wang, J.C. (2002) Nat. Rev. Mol. Cell Biol. 3, 430-440

23 Wu, L. and Hickson, I.D. (2003) Nature (London) 426, 870-874

24 Wu, L., Chan, K.L., Ralf, C., Bernstein, D.A., Garcia, P.L., Bohr, V.A., Vindigni, A., Janscak, P., Keck, J.L. and Hickson, I.D. (2005) EMBO J. 24, 2679-2687

Received 26 June 2005 